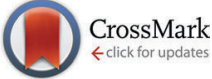

Cite this: Phys. Chem. Chem. Phys., 2016, 18, 27342

Received 18th April 2016, Accepted 14th September 2016 DOI: $10.1039 / c 6 c p 02575 g$ www.rsc.org/pccp

\title{
Magnetomigration of rare-earth ions in inhomogeneous magnetic fields $\dagger$
}

\author{
Agnieszka Franczak, ${ }^{a}$ Koen Binnemans ${ }^{b}$ and Jan Fransaer*a
}

\begin{abstract}
The effects of external inhomogenous (gradient) magnetic fields on the movement of the rare-earth ions: $\mathrm{Dy}^{3+}, \mathrm{Gd}^{3+}$ and $\mathrm{Y}^{3+}$, in initially homogeneous aqueous solutions have been investigated. Differences in the migration of rare-earth ions in gradient magnetic fields were observed, depending on the magnetic character of the ions: paramagnetic ions of $\mathrm{Dy}^{3+}$ and $\mathrm{Gd}^{3+}$ move towards regions of the sample where the magnetic field gradient is the strongest, while diamagnetic ions of $\mathrm{Y}^{3+}$ move in the opposite direction. It has been showed that the low magnetic field gradients, such the ones generated by permanent magnets, are sufficient to observe the magnetomigration effects of the ions in solution. The present work clearly establishes the behavior of magnetically different ions in initially homogeneous aqueous solutions exposed to magnetic field gradients. To this avail, a methodology for measuring the local concentration differences of metal ions in liquid samples was developed.
\end{abstract}

\section{Introduction}

It is well-known that an external inhomogeneous magnetic field can induce transport of ions and molecules in solution. ${ }^{1-6}$ This magnetomigration of ions and molecules is observed in inhomogeneous (gradient) magnetic fields in which the position of species in solution depends on the magnetic field strength at each point. In the presence of an inhomogeneous magnetic field, the magnetic field on an ion immersed in a solution is the magnetic field gradient force (Kelvin force), which can be written as: $:^{7-15}$

$$
\vec{F}=\frac{\chi_{\text {ion }}-\chi_{\text {sol }}}{\mu_{0}} V_{\text {ion }}(\vec{B} \cdot \vec{\nabla}) \vec{B}
$$

where $\chi_{\text {ion }}$ and $\chi_{\text {sol }}$ are the dimensionless magnetic susceptibilities of the ion and the solution [-], respectively; $V_{\text {ion }}$ is the volume of the ion $\left[\mathrm{m}^{3}\right], \vec{B}$ is the magnetic field $[\mathrm{T}], \nabla \vec{B}$ is the magnetic field gradient $\left[\mathrm{T} \mathrm{m}^{-1}\right]$ and $\mu_{0}$ is the magnetic permeability of vacuum $\left[4 \pi 10^{-7} \mathrm{H} \mathrm{m}^{-1}\right]$.

The magnitude of the magnetic forces acting on species in solution is determined by the magnetic character of metal ions and molecules which can be either paramagnetic or diamagnetic. Paramagnetic species are characterized by the presence of unpaired electrons in atomic or molecular orbitals. ${ }^{16}$ Electrons have spins that can be considered as permanent magnetic dipole moments.

\footnotetext{
${ }^{a}$ Department of Materials Engineering, KU Leuven, Kasteelpark Arenberg 44, 3001 Heverlee, Belgium. E-mail: Jan.Fransaer@mtm.kuleuven.be

${ }^{b}$ Department of Chemistry, KU Leuven, Celestijnenlaan 200 F, 3001 Heverlee, Belgium

$\dagger$ Electronic supplementary information (ESI) available. See DOI: 10.1039/ c6cp02575g
}

In the absence of an external magnetic field the magnetic dipole moments are randomly oriented, cancelling each other on a macroscopic scale. When an external magnetic field is applied, the magnetic dipole moments of the paramagnetic species become aligned with the lines of the magnetic field flux, leading to a net magnetization: ${ }^{14,17}$

$$
M=\chi H
$$

where $\chi$ is the dimensionless Curie-law magnetic susceptibility [-] and $H$ the applied magnetic field [ $\mathrm{A} \mathrm{m}^{-1}$. However, the magnetic dipole moments are only partially oriented because the magnetic alignment has to compete with the thermal motion existing at all the times in a sample. On the contrary, diamagnetic species possess only paired electrons in atomic and molecular orbitals and no magnetization can be induced. ${ }^{16}$ However, an external magnetic field causes small distortions of the electron orbits within molecules. Magnetic dipole moments are induced and aligned in a direction opposite to the magnetic field flux lines. The existence of dipole magnetic moments in diamagnetic species can be observed, but only under external magnetic field and the magnetic moments retain in a sample as long as the magnetic field is applied. Hence, under gradient magnetic field conditions paramagnetic metal ions and molecules present in solution will migrate in the direction of increasing magnetic field, while diamagnetic species will be repelled by the magnetic field and migrate towards regions with weaker magnetic field strengths. ${ }^{18}$ However, recent studies suggest that a magnetic gradient force is not likely to have any effect on ions and molecules due to the huge difference between the kinetic and magnetic energies of the species in solution. ${ }^{15}$ 
The theory of Brownian motion predicts that the smaller the particle size the larger its mean-squared displacement. The energy carried by the magnetic field is much lower than $k_{\mathrm{B}} T$ at room temperature $\left(k_{\mathrm{B}}\right.$ being the Boltzmann constant and $T$ the absolute temperature) and hence, any form of magnetic migration is eradicated by the thermal noise. ${ }^{7}$ Moreover, even if an excited state was induced in an ion by a magnetic field, it should rapidly disappear as a result of the thermal collisions when the magnetic field is removed. ${ }^{19,20}$ Nevertheless, remarkable effects have recently been observed for aqueous solutions in external magnetic fields. ${ }^{21-24}$ Schadewal et al. ${ }^{7}$ investigated the influence of a magnetic gradient force on the distribution of dissolved paramagnetic $\mathrm{Fe}^{2+}$ ions at different concentrations, directions of the magnetic field gradient and magnetic field strength. Experiments on solutions consisting of paramagnetic $\mathrm{Fe}^{2+}$ ions showed a significant increase in the $\mathrm{Fe}^{2+}$ ions concentration when a magnetic field gradient was applied. The effect of gradient magnetic fields on different paramagnetic transition metal ions was also studied by Fujiwara et al. ${ }^{25-28}$ Paramagnetic ions, such as $\mathrm{Fe}^{3+}, \mathrm{Co}^{2+}, \mathrm{Ni}^{2+}$ and $\mathrm{Cu}^{2+}$ were spotted on a silica gel support. The ions moved in the direction of the strongest gradient magnetic fields. The authors showed that the extent of displacement of ion-containing silica particles depended on the magnetic susceptibility of the ions, their adsorption properties on the silica particles and their concentration. Yang et al. ${ }^{29-31}$ demonstrated that paramagnetic $\mathrm{Mn}^{2+}$ and $\mathrm{Dy}^{3+}$ ions in a homogeneous aqueous solution can be locally enriched by means of a superimposed gradient magnetic field and this enrichment depended on the magnetic susceptibility of the considered metal ions. Because the effects of an external magnetic field on species in the liquid samples are rather difficult to observe due to the occurrence of a natural convection when the magnetic field is removed, Yang et al. ${ }^{30,31}$ and Uhlemann et al. ${ }^{9}$ used a Mach-Zehnder interferometry to investigate the concentration gradient of paramagnetic metal ions in initially homogeneous solutions caused by the application of a magnetic field generated by $\mathrm{NdFeB}$ magnets. This method allows for observation of the concentration changes of metal ions in solution caused by an inhomogeneous magnetic field, but it is an indirect method and it cannot be used for observation of the liquid samples in the bore of a superconducting magnet.

Most of the studies on magnetomigration of metal ions in inhomogeneous magnetic fields were about d-block transition metals. Nevertheless, the magnetic field effects on rare-earth ions are of special interest due to their unique magnetic properties. The rare-earth ions with unpaired electrons are characterized by a large unquenched orbital angular momentum associated with the internal nature of the valence $4 \mathrm{f}$ orbitals. With the exception of $\mathrm{Gd}^{3+}$ and $\mathrm{Eu}^{2+}$, which have a $4 \mathrm{f}^{7}$ electronic configuration and an orbitally non-degenerate ground state, and $\mathrm{Sc}^{3+}, \mathrm{Y}^{3+}, \mathrm{La}^{3+}$ and $\mathrm{Lu}^{3+}$ which have an empty or full $4 \mathrm{f}$ shell, all other rare-earth ions possess orbitally degenerated ground states, obtained from the following interactions, given in the decreasing order of their relative strength: the interelectronic repulsion, the spin-orbit coupling and the crystal-field effects. ${ }^{32}$
Since crystal-field effects are smaller and spin-orbit coupling larger for $\mathrm{f}$ electrons in comparison with the $\mathrm{d}$ electrons of transition metal ions, the orbital component of the magnetic moment is larger for the rare-earth ions than for the transition metal ions. This is due to the larger nuclear charge of the rare-earth ions. At the same time, the crystal-field of rare-earth ions is much smaller than for d electrons, since the $4 \mathrm{f}$ orbitals are compressed and the $4 \mathrm{f}$ electrons are screened by the filled outer $5 \mathrm{~s}$ and $5 \mathrm{p}$ shells. ${ }^{33}$ Hence, paramagnetic rare-earth ions have unique magnetic properties compared to d-block transition metal ions. However, very few studies have been devoted to the magnetomigration of paramagnetic rare-earth ions in magnetic field gradients. ${ }^{30,34,35}$

In this paper, the effect of external inhomogeneous magnetic fields on the migration of paramagnetic and diamagnetic rare-earth ions is described. For the first time, the magnetomigration of diamagnetic rare-earth ions is reported. These investigations show the development of concentration gradients due to the migration of rare-earth ions in an initially homogeneous solution under applied external inhomogeneous magnetic fields. A novel experimental method is described for the direct measurement of concentration gradients of metal ions caused by an external magnetic field.

\section{Experimental}

\section{Materials}

Dy $\left(\mathrm{NO}_{3}\right)_{3} \cdot x \mathrm{H}_{2} \mathrm{O}$ (Aldrich, 99.9\%), $\mathrm{Y}\left(\mathrm{NO}_{3}\right)_{3} \cdot 6 \mathrm{H}_{2} \mathrm{O}$ (Alfa Aesar, 99.9\%), $\mathrm{Gd}\left(\mathrm{NO}_{3}\right)_{3} \cdot 6 \mathrm{H}_{2} \mathrm{O}$ (Sigma-Aldrich, 99.9\%).

\section{Methods}

Gel samples. Individual solutions of $\mathrm{Dy}^{3+}(3 \mathrm{mM}), \mathrm{Y}^{3+}(3 \mathrm{mM})$ and $\mathrm{Gd}^{3+}(3 \mathrm{mM})$ were obtained by dissolving the corresponding nitrate salts in demineralized water. In order to avoid the hydrolysis of the rare-earth ions, the $\mathrm{pH}$ of the solutions was adjusted to 3 by addition of $65 \% \mathrm{HNO}_{3}$. The resulted solutions were transferred to $50 \mathrm{~mL}$ glass beakers. The solutions were then heated up to $40^{\circ} \mathrm{C}$ on a hot plate and $8 \mathrm{wt} \%$ of gelatin was added. The mixture was stirred until complete dissolution of the gelatin powder and then cooled to $25{ }^{\circ} \mathrm{C}$. In the meanwhile, glass tubes $(4.5 \mathrm{~cm}$ in length and inner diameter of $1.2 \mathrm{~cm}$ ) were prepared: the inner walls of tubes were coated with a thin layer of grease. Then, one end of the tube was covered by a sheet of Parafilm and placed at the bottom of a vessel filled with ice water. The cooled mixture of gelatin and the rare-earth ions was poured inside the glass tubes that were then covered with a sheet of Parafilm. The low temperature of the bath allowed for immediate (about $2 \mathrm{~s}$ ) gelling of the samples. In order to achieve a stable gel, the prepared samples were kept overnight in a fridge at $6{ }^{\circ} \mathrm{C}$. Before each experiment, the gelled samples were taken out of the fridge and left at room temperature for $1 \mathrm{~h}$. Each solution was prepared individually according to this procedure. The obtained gel samples were then prepared for the magnetic experiments: the Parafilm covers were removed from the glass tube and the gel sample was pushed 


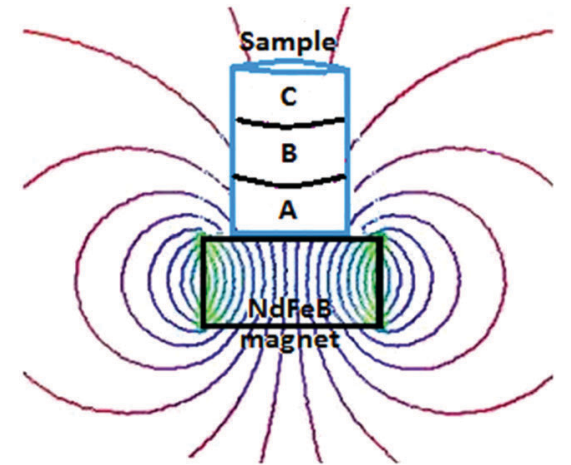

Fig. 1 Schematic view of the experimental set-up with the sample on top of the NdFeB permanent magnet. A, B, C indicate the positions of the gel slices or the porous disks used for analysis of the metal content.

out the tube using a micrometer. The top and bottom part of the gel samples were adjusted to a total height of $6 \mathrm{~mm}$ using a thin wire cutter. The as-prepared samples were put on the top of the permanent magnets (Fig. 1) or into the bore of a superconducting magnet for a certain period of time. After exposure to the magnetic field, the $6 \mathrm{~mm}$ long gel samples were pushed out off the glass tubes by a micrometer and divided into three pieces, each $2 \mathrm{~mm}$ in height. The gel pieces were transferred into separate flasks and $3 \mathrm{~mL}$ of demineralized water was added. The flasks were heated on a hot plate, re-dissolving the gel pieces. The resulting samples were transferred to $100 \mathrm{~mL}$ volumetric flasks for chemical analysis.

Liquid samples. Individual solutions of $\mathrm{Dy}^{3+}(4 \mathrm{mM})$ and $\mathrm{Y}^{3+}$ $(10 \mathrm{mM})$ were obtained by dissolving the corresponding nitrate salts in demineralized water. The acidity of the solutions was adjusted to $\mathrm{pH}=3$ by addition of $65 \% \mathrm{HNO}_{3}$. In this approach, the porous glass disks $(2 \mathrm{~cm}$ in diameter and $2 \mathrm{~mm}$ in thickness, $40-100 \mu \mathrm{m}$ pore size) were used. At first, $10 \mathrm{~mL}$ of solution was pushed through the membranes using a vacuum pump. The porous glass disks were then immersed into solutions containing individual rare-earth ions for duration of $24 \mathrm{~h}$. The solutions were additionally stirred from the top at all times by a mechanical agitator.

The silicone tubes $(2 \mathrm{~cm}$ in diameter and $6 \mathrm{~cm}$ in length) were used as sample holders. Each holder contained three porous glass disks, saturated with the rare-earth solutions. The disks were pushed closely together and both tube ends were covered by a sheet of Parafilm. The as-prepared samples were placed on top of a permanent magnet (Fig. 1). After exposure to the magnetic field, the rare-earth ions were extracted from the porous glass disks into $100 \mathrm{~mL}$ volumetric flasks using a vacuum pump.

Magnetic field sources. The magnetic fields were generated by the $\mathrm{N} 52$ neodymium-iron-boron (NdFeB) permanent magnets (Supermagnete, Germany) or a superconducting magnet (Cryomagnetics, Inc., USA). The superconducting magnet had a solenoid vertical uniform magnetic field up to $10 \mathrm{~T}$ and a bore diameter of $100 \mathrm{~mm}$. The permanent magnets had a rectangular shape with dimensions of $50 \times 50 \times 25 \mathrm{~mm}$. The expected magnetic field generated by the $\mathrm{N} 52 \mathrm{NdFeB}$ permanent magnets is 1.26 to $1.29 \mathrm{~T}$. However, these reported values are the so-called residual magnetism which is the magnetization in the core of the magnet that is not exposed to an external magnetic field. Thus, in order to obtain the real magnetic field intensity at the magnet surface, the actual magnetic field strength was measured (see ESI $\dagger$ ).

Magnetic susceptibility measurements. The magnetic susceptibilities of the gel samples were measured by a Gouy method at room temperature. The magnetic field of $0.534 \mathrm{~T}$ was generated by an electromagnet. The gel sample was uniformly packed into a glass tube $\left(0.08 \mathrm{~cm}^{2}\right.$ cross section), which was suspended vertically from a microbalance between the two pole faces of an electromagnet. The bottom of the sample was leveled with the center of the pole faces, so the sample could be exposed to an inhomogeneous magnetic field, along the vertical axis. The mass changes of the gel samples were recorded after applying the external magnetic field. Triplicate measurements were done for each sample.

Concentration analysis. Inductively coupled plasma optical emission spectrometry (ICP-OES) was used to determine the concentration changes in the experimental samples. Triplicate concentration analyses were done for each sample. The ICP-OES measurements were carried out using a Varian 720 ES spectrometer. The dynamic ranges for the ICP-OES were $0.003-150 \mathrm{ppm}$ for $\mathrm{Dy}^{3+}, 0.002-100 \mathrm{ppm}$ for $\mathrm{Y}^{3+}$ and $0.025-1250 \mathrm{ppm}$ for $\mathrm{Gd}^{3+}$. Thus, the samples were diluted to be within these concentration ranges and standard solutions were prepared from $1000 \mathrm{ppm}$ standard solutions of the considered ions. The concentrations of considered ions were measured at $353.17 \mathrm{~nm}$ for $\mathrm{Dy}^{3+}$, $377.43 \mathrm{~nm}$ for $\mathrm{Y}^{3+}$ and $342.246 \mathrm{~nm}$ for $\mathrm{Gd}^{3+}$.

\section{Results}

The following section discusses two different techniques to study the effect of magnetic field gradients on rare-earth ions: gel samples and porous glass disks. Both experimental approaches prevent natural convection and allow for the chemical analysis of the samples as a function of position after removal of the magnetic field.

Two solutions containing paramagnetic ions, one with $\mathrm{Dy}^{3+}$ ions $(3 \mathrm{mM})$ and another with $\mathrm{Gd}^{3+}$ ions $(3 \mathrm{mM})$, and one solution containing diamagnetic $\mathrm{Y}^{3+}$ ions $(3 \mathrm{mM})$ were gelled using gelatin. After achieving a full state of gelling, a stable gel sample containing rare-earth ions was obtained. The as-prepared gel sample in the glass holder was then placed on top of a NdFeB permanent magnet or in the bore of a superconducting magnet for a certain period of time.

After exposure to the magnetic field, the gel was pushed out of the holder by a micrometer so that the thickness of the extracted pieces could be controlled. In this way, three samples were obtained: slice A $-0-2 \mathrm{~mm}$ from the point of the strongest magnetic field gradient, slice B $-2-4 \mathrm{~mm}$, and slice C $-4-6 \mathrm{~mm}$. In accordance with the variation in magnetic field strength as a function of the distance from the magnet surface (see ESI $\dagger$ ) each slice of the gel sample was exposed to a magnetic field of a 

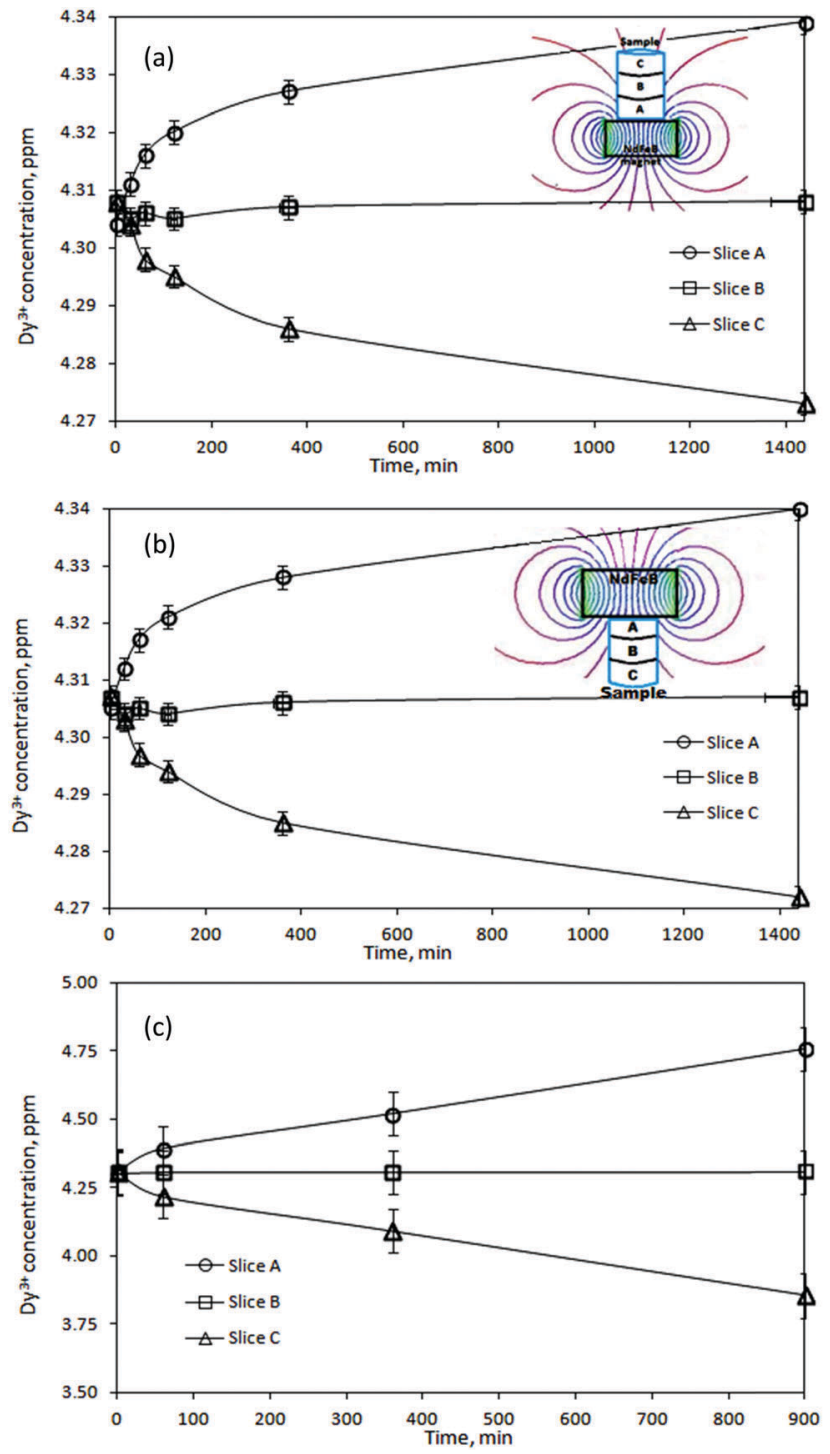

Fig. 2 ( $a$ and b) Concentration changes of $\mathrm{Dy}^{3+}$ ions induced by a NdFeB permanent magnet $\left(B=0.5 \mathrm{~T}, B \mathrm{~dB} / \mathrm{dz}=14.5 \mathrm{~T}^{2} \mathrm{~m}^{-1}\right)$, and (c) by a superconducting magnet $\left(B=5 \mathrm{~T}, B \mathrm{~d} B / \mathrm{dz}=189 \mathrm{~T}^{2} \mathrm{~m}^{-1}\right)$ in gel samples. Slices $A$, $B$ and $C$ are the distances from the strongest magnetic field gradient point: slice A: 0-2 mm, slice B: 2-4 mm and slice C: 4-6 mm.

different strength. Therefore, the concentration of the rareearth ions was expected to vary from slice to slice. To verify this, the slices were re-dissolved and their concentrations were determined by ICP-OES. Fig. 2a shows the results for the gel samples containing $\mathrm{Dy}^{3+}$ ions exposed to the magnetic field generated by a NdFeB permanent magnet. It can be seen that in the initially homogeneous samples of rare-earth ions exposed to a magnetic field gradient, a concentration gradient was induced. The concentration of paramagnetic $\mathrm{Dy}^{3+}$ ions increases in the vicinity of the magnet (0-2 mm, slice A). Thus, the concentration of the paramagnetic $\mathrm{Dy}^{3+}$ ions was enriched in the part of the sample where the magnetic field gradient was the strongest. In Fig. 2a, the enrichment of $\mathrm{Dy}^{3+}$ ions progresses relatively rapidly up to an exposure time of $180 \mathrm{~min}$ to the magnetic field and then slows down. However, the steady state had not been achieved yet and the concentration variations of the $\mathrm{Dy}^{3+}$ ions were still observed after $1440 \mathrm{~min}$. After this time, an enrichment of $0.8 \%$ with respect to the initial concentration was achieved in slice A. Fig. $2 \mathrm{~b}$ indicates similar results when changing the position of the permanent magnet: from the bottom of the glass tube to the top of the tube. In order to verify a dependence of the induced concentration gradient on the applied magnetic field gradient, the gel samples containing paramagnetic $\mathrm{Dy}^{3+}$ ions were exposed to the high magnetic field, $B=5 \mathrm{~T}\left(B \mathrm{~d} B / \mathrm{d} z=189 \mathrm{~T}^{2} \mathrm{~m}^{-1}\right)$, generated by a superconducting magnet (Fig. $2 \mathrm{c}$ ). In this case, the $\mathrm{Dy}^{3+}$ gel samples exposed to the high magnetic field gradient during 900 min resulted in the enrichment of $\mathrm{Dy}^{3+}$ ions in slice A of about $10 \%$ with respect to the initial concentration. Similar to the previous investigation concerning the low magnetic field gradient, also at high magnetic field gradient the concentration steady state had not been achieved even $900 \mathrm{~min}$ after applying the magnetic field. Comparing both results, from low and high magnetic field gradients (Fig. 3), it can be clearly seen that increasing an external magnetic field by 10 fold the enrichment of $\mathrm{Dy}^{3+}$ ions in slice A also significantly increases: by 7 fold after magnetic field exposure during $60 \mathrm{~min}$ and 9 fold after magnetic field exposure during $360 \mathrm{~min}$. Similar observations were noticed when using agarose or agar-agar as gelating agents.

To confirm that the observed magneto-induced migration of the paramagnetic $\mathrm{Dy}^{3+}$ ions is caused by an external magnetic field, the magnetic susceptibilities of the gel samples were investigated. The magnetic susceptibility measurements were done for the $\mathrm{Dy}^{3+}$ gel samples exposed to the low magnetic field gradient generated by a $\mathrm{NdFeB}$ permanent magnet for various periods of time. Each of the slices extracted after exposure of the gel samples to the magnetic field was closely packed into a glass tube with kept weight control of the sample. The glass tube was then placed between the pole faces of an electromagnet in a way that the tube was exposed to the inhomogeneous magnetic field. The mass changes of the sample after applying the magnetic field were recorded and mass magnetic susceptibility calculated. Fig. 4 shows the average magnetic susceptibilities (after triplicate measurements) of the $\mathrm{Dy}^{3+}$ gel samples as a

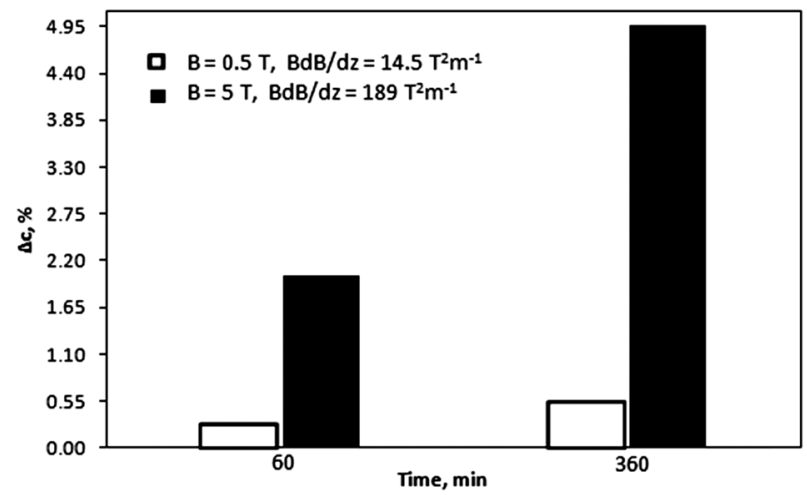

Fig. 3 Effect of a magnetic field on the induced concentration gradient in the $\mathrm{Dy}^{3+}$ gel samples (slice A) as a function of time of an applied magnetic field. 


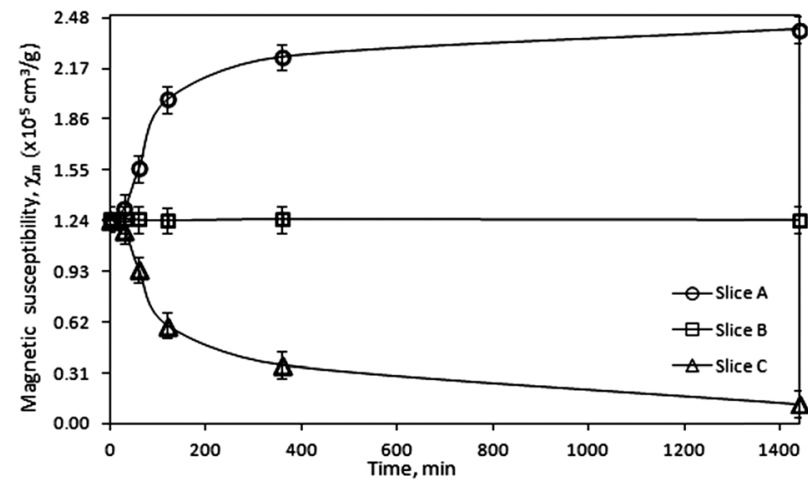

Fig. 4 Magnetic susceptibility, $\chi_{m}$, of the $D y^{3+}$ gel samples after exposure to the magnetic field generated by a NdFeB permanent magnet $(B=0.5 \mathrm{~T}$, $B d B / d z=14.5 T^{2} \mathrm{~m}^{-1}$.

function of time of the exposure to the magnetic field. It can be seen that the magnetic susceptibility follows the trend of the ion concentration changes (Fig. 2a and b) caused by the presence of an external magnetic field. The magnetomigration of the paramagnetic $\mathrm{Dy}^{3+}$ ions towards the region of the sample where the magnetic field gradient was the strongest (slice A) resulted in a higher concentration of the ions in this region what in consequence reflects in the increased magnetic susceptibility. Thus, the observed concentration gradient formed in the $\mathrm{Dy}^{3+}$ gel samples is definitely caused by the applied external magnetic field.

Further confirmation of the magnetic field effects on the migration of ions in solution were investigated by studying the reverse concentration changes when the magnetic field was removed. The $\mathrm{Dy}^{3+}$ gel samples were first exposed to an external magnetic field for $1440 \mathrm{~min}$, after which the magnetic field was removed for various periods of time. The gel samples were extracted in the same way as during magnetic measurements, dividing the gel sample for three identical slices. Fig. 5a shows the $\mathrm{Dy}^{3+}$ concentration changes in the gel after removal of the magnetic field as a function of time. Due to the diffusion of ions in the gel sample, the concentration gradient of $\mathrm{Dy}^{3+}$ ions induced earlier by a magnetic field is lost as ions start to migrate from regions of high concentration to regions of low concentration. As a consequence, $120 \mathrm{~min}$ after removal of the magnetic field, the concentration of ions in each gel slice was the same as in the reference sample, before exposing it to the external magnetic field. Additional confirmation of the effect of a magnetic field is the concentration analysis of a reference sample, which was not exposed to an external magnetic field (Fig. 5b). It was observed that the ion distribution in the gel samples was uniform and did not change over time. This indicates that the gel matrix did not influence the experimental results, but just impeded the natural convection in the sample. This also indicates that the magnetic field causes the concentration differences in the gel in the first place.

Next, the paramagnetic $\mathrm{Gd}^{3+}$ ions were studied. Fig. 6a shows a concentration increase of $0.6 \%$ with respect to the initial concentration, in the region of the sample where the magnetic
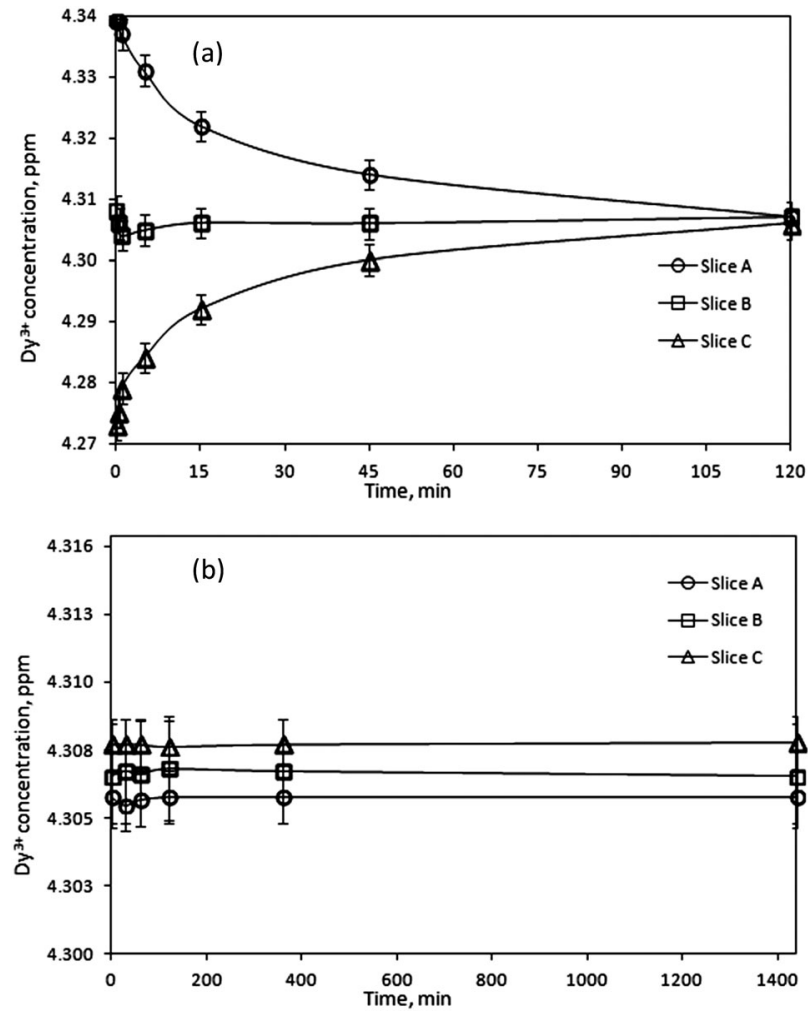

Fig. 5 (a) Concentration changes of $D y^{3+}$ ions after removal of the magnetic field gradient ( $\mathrm{NdFeB}$ permanent magnet, $B=0.5 \mathrm{~T}, B \mathrm{~d} B / \mathrm{d} z=$ $14.5 \mathrm{~T}^{2} \mathrm{~m}^{-1}$ ); (b) reference sample without the presence of a magnetic field. Slices $A, B$ and $C$ are the distances from the strongest magnetic field gradient point: slice A: 0-2 mm, slice B: 2-4 mm and slice C: $4-6 \mathrm{~mm}$.

field gradient was the strongest (slice A), after $1440 \mathrm{~min}$ of exposure to the magnetic field. The fact that $\mathrm{Gd}^{3+}$ behaves similarly to the paramagnetic $\mathrm{Dy}^{3+}$ ions under external magnetic field indicates that the observed magnetomigration of paramagnetic rare earths ions is governed by the magnetic moment, unlike the magnetic alignment of rare-earth-containing liquid crystals where the anisotropic and isotropic characters of the rare-earths had to be taken into account. ${ }^{34}$

However, the behavior of the diamagnetic $\mathrm{Y}^{3+}$ ions was opposite to that of the paramagnetic rare-earth ions and in this case the concentration decreased in the region closest to the magnet surface ( $0-2 \mathrm{~mm}$, slice A) (Fig. 6b). ICP analysis indicated a decrease of $0.6 \%$ in slice $\mathrm{A}$ compared to the initial $\mathrm{Y}^{3+}$ concentration. Thus, $\mathrm{Y}^{3+}$ ions are repelled by the magnetic field. Similarly to the $\mathrm{Dy}^{3+}$ and $\mathrm{Gd}^{3+}$ ions, a steady state concentration profile of $\mathrm{Y}^{3+}$ ions was not obtained after $180 \mathrm{~min}$, and the concentration increase of diamagnetic ions at the other side of the sample (4-6 mm, slice C) where the magnetic field strength was the lowest, continues even after 1440 min.

To unequivocally exclude the influence of the gelatin matrix on the observed magnetic field effects on the different rare-earth ions, a second approach based on the use of pure solutions soaked in porous glass disks (without gelatin) was developed. Before each experiment, the porous glass disks were immersed in solutions of $\mathrm{Dy}^{3+}(4 \mathrm{mM})$ or $\mathrm{Y}^{3+}(10 \mathrm{mM})$. The principle of the 

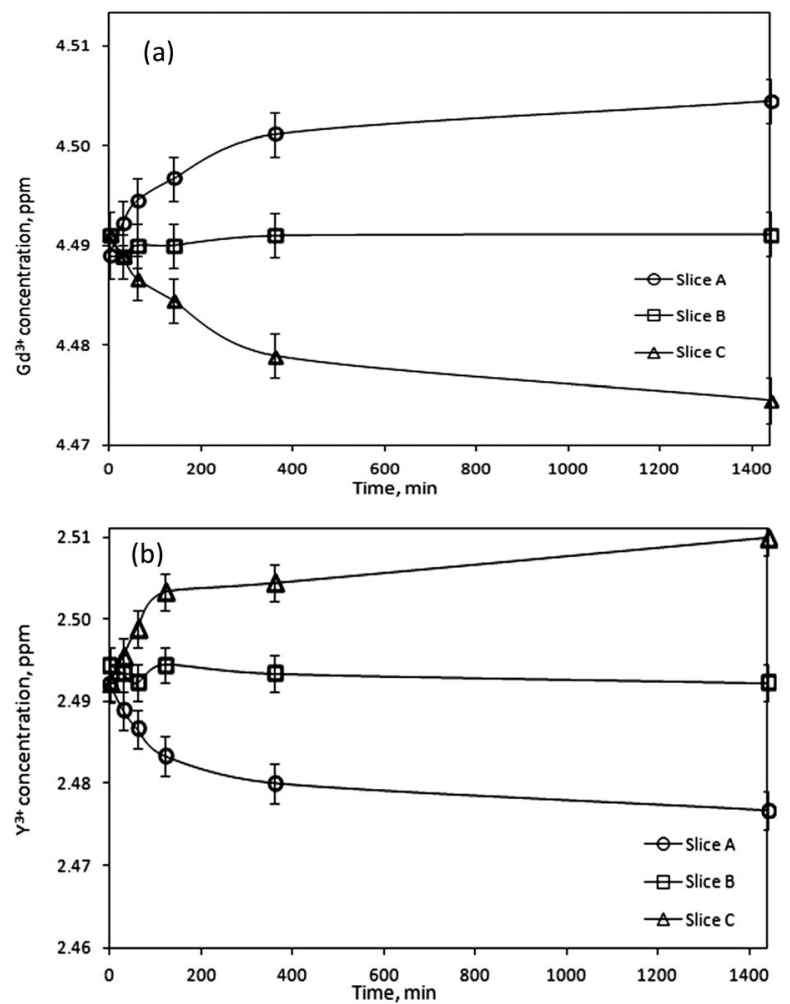

Fig. 6 Concentration changes of (a) $\mathrm{Gd}^{3+}$ ions and (b) $\mathrm{Y}^{3+}$ ions induced by a NdFeB permanent magnet in gel samples. Slices $A, B$ and $C$ are the distances from the strongest magnetic field gradient point: slice $A: 0-2 \mathrm{~mm}$, slice B: 2-4 $\mathrm{mm}$ and slice C: $4-6 \mathrm{~mm}$.

experimental setup was similar to the one with the gelatin matrix. Thus, samples consisting of three small glass disks, each $2 \mathrm{~mm}$ thick, were placed on top of a permanent magnet for a certain period of time. The magnetic exposure time had to be kept much shorter compared to the experiments with the gel samples due to evaporation of the solution. After exposure to the magnetic field, the samples were extracted and the concentration of the rare-earth ions was measured. Fig. 7 shows a similar result to the one observed in the gel samples: the paramagnetic $\mathrm{Dy}^{3+}$ ions moved to the region of the strongest magnetic field localized at the magnet surface (slice $\mathrm{A}$ ), while the diamagnetic $\mathrm{Y}^{3+}$ ions were repelled and a concentration decrease in the region of the strongest field (slice A) was observed. Experiments on solutions of magnetically different rare-earth ions indicate a $6 \%$ increase in concentration of $\mathrm{Dy}^{3+}$ ions and a $7 \%$ decrease of $\mathrm{Y}^{3+}$ ions in the strongest magnetic field. However, the concentration changes happen more quickly in porous glass than in gel samples: after $360 \mathrm{~min}$ the concentration profile in the glass disks has attained its steady state while in gel samples, the concentration has still not attained its steady state after $1440 \mathrm{~min}$. The faster and larger differences found for the porous glass samples compared to the gel samples are due to the differences in the matrices. In the gelatin matrix, the ions have to move through the interchain voids among the gel fibers, which are of nanometer size. The pore size of the glass disks is much larger, between 40 and $100 \mu \mathrm{m}$. Therefore, the motion of ions is
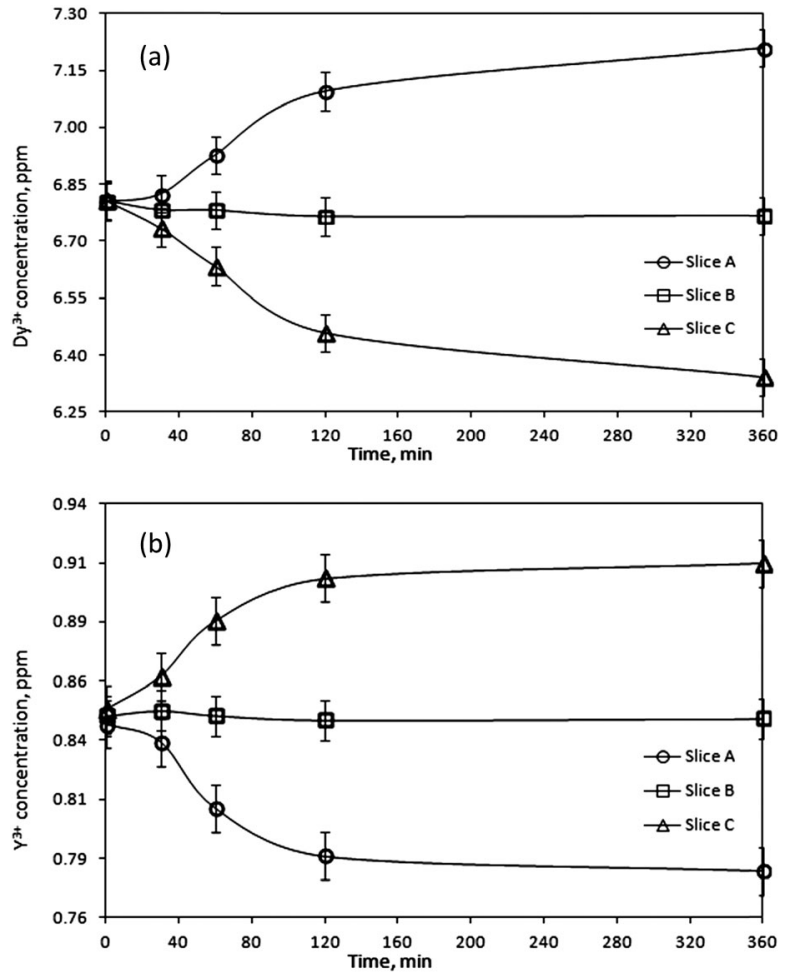

Fig. 7 Concentration changes of (a) $D y^{3+}$ ions and (b) $\mathrm{Y}^{3+}$ ions induced by a NdFeB permanent magnet in porous glass disks as a function of exposure time to the magnetic field gradient. Slices $A, B$ and $C$ are the distances from the strongest magnetic field gradient point: slice $A: 0-2 \mathrm{~mm}$, slice $B$ : 2-4 $\mathrm{mm}$ and slice C: $4-6 \mathrm{~mm}$.

less disturbed by interactions with the matrix, increasing their rate of diffusion.

\section{Discussion}

The present work clearly establishes the migration of magnetically different ions in initially homogeneous aqueous solutions exposed to magnetic field gradients. Fig. 8 shows the relative change in the concentration of $\mathrm{Dy}^{3+}$ ions subject to magnetic field gradients of increasing strength compared to the initial $\mathrm{Dy}^{3+}$ concentration. This figure also shows that the concentration difference increases when the magnetic field gradient increases. In steady state, the spatial distribution of the ion concentration, $c=c(x)$, in a one-dimensional system subjected to a magnetic field gradient, $\nabla B$ at temperature $T$, should be proportional to the Boltzmann factor: ${ }^{36}$

$$
c(x)=c \cdot \exp \left(\frac{\chi_{\text {ion }}-\chi_{\text {sol }}}{2 k_{\mathrm{B}} T} B(x)^{2}\right)
$$

where

$$
\chi_{\text {ion }}=\frac{\mu_{\text {eff }}^{2}}{3 k_{\mathrm{B}} T}
$$

However, if we scale $\Delta c$ by $\exp \left(\frac{\chi_{\text {ion }}-\chi_{\text {sol }}}{2 k_{\mathrm{B}} T} B(x)^{2}\right)$, the data of Fig. 8 do not fall on a straight line. Moreover, when a magnetic field 


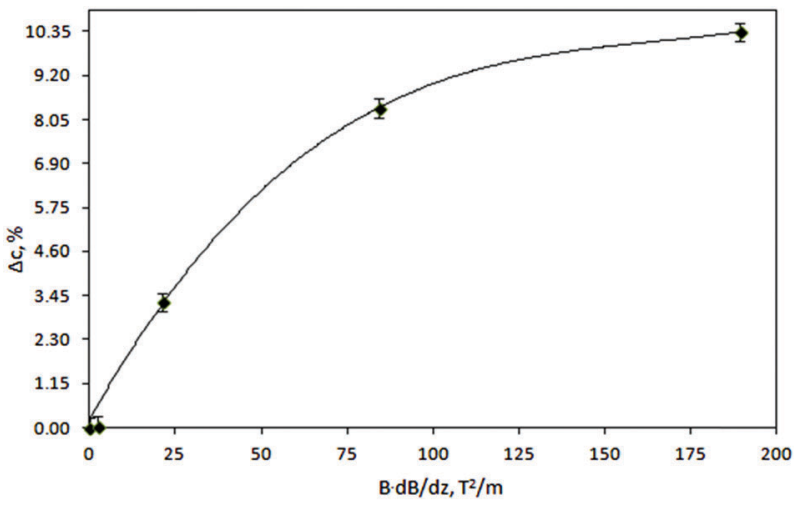

Fig. 8 Difference between the concentration of $\mathrm{Dy}^{3+}$ ions in slice $\mathrm{A}$ $(0-2 \mathrm{~mm}$ from the strongest magnetic field times magnetic field gradient point) and the initial concentration in the gel samples after $900 \mathrm{~min}$ of exposure to the magnetic field, as a function of the product of the magnetic field times the magnetic field gradient.

of $10 \mathrm{~T}$ is applied to a solution of $\mathrm{Dy}^{3+}$ ions for which $\mu_{\mathrm{eff}}$ is 10.63 $\mu_{\mathrm{B}}\left(\mu_{\mathrm{B}}=\frac{e \hbar}{2 m_{\mathrm{e}}}=9.27401 \times 10^{-24} \mathrm{~J} \mathrm{~T}^{-1}\right.$ is the Bohr magneton $)$ at $300 \mathrm{~K}$, the induced concentration change should be equal to: ${ }^{36}$

$$
\Delta c=\frac{c(10 \mathrm{~T})-c(0 \mathrm{~T})}{c(0 \mathrm{~T})}=0.97 \%
$$

Nevertheless, the concentration changes induced in the $\mathrm{Dy}^{3+}$ samples by a magnetic field of already $5 \mathrm{~T}$ imposed during $900 \mathrm{~min}$ is of the order of $10 \%$, what is roughly 10 fold larger than the calculated value for the field of $10 \mathrm{~T}$.

Moreover, considering differences in the magnetic moments of the studied ions, the solution of $\mathrm{Y}^{3+}$ ions, with $\mu_{\text {eff }}=0 \mu_{\mathrm{B}}$, under the same experimental conditions should not show any concentration variation, which is at odds with the results shown in Fig. 6b. A similar effect of a magnetic field gradient on diamagnetic ions was also reported by Yang et al. ${ }^{31}$ when investigating dilute $\mathrm{CuSO}_{4}$ solution: in the vicinity of the strongest magnetic field gradient, a concentration reduction up to $\Delta c \sim-0.7 \mathrm{mM}$ was observed.

Also, no scaling is observed with the magnetic susceptibility of the ions. This is shown in Fig. 9 where the change in the concentration of $\mathrm{Dy}^{3+}, \mathrm{Gd}^{3+}$ and $\mathrm{Y}^{3+}$ ions and their initial concentration is plotted as a function of $\Delta \chi=\chi_{\text {ion }}-\chi_{\text {sol }}$, the difference between the dimensionless ([-]) magnetic susceptibilities of the ions and water $\left(\chi_{\mathrm{H}_{2} \mathrm{O}}:-0.16 \times 10^{-3}[-] ; \chi_{\mathrm{Dy}^{3+}}\right.$ : $+1.23[-], \chi_{\mathrm{Gd}^{3+}}:+2.32[-]$ and $\left.\chi_{\mathrm{Y}^{3+}}:+2.3 \times 10^{-3}[-]\right){ }^{36-39}$ This figure shows that the slightly diamagnetic $\mathrm{Y}^{3+}$ ions migrate almost as much under the same circumstances as the strongly paramagnetic $\mathrm{Dy}^{3+}$ and $\mathrm{Gd}^{3+}$ ions. Pulko et al. ${ }^{30}$ reported similar observation when comparing the concentration gradients of $\mathrm{Dy}^{3+}$ ions to $\mathrm{Mn}^{2+}$ ions, studied earlier by Yang et al. ${ }^{31}$ In this case, the magnetic field gradient force should be larger for $\mathrm{Dy}^{3+}$ ions because the magnetic susceptibility of $\mathrm{Dy}^{3+}$ exceeds that of $\mathrm{Mn}^{2+}$. Thus, the concentration gradient of $\mathrm{Dy}^{3+}$ ions should be both higher and reached faster than in the case of $\mathrm{Mn}^{2+}$ ions. However, this hypothesis was in contrast with the experimental

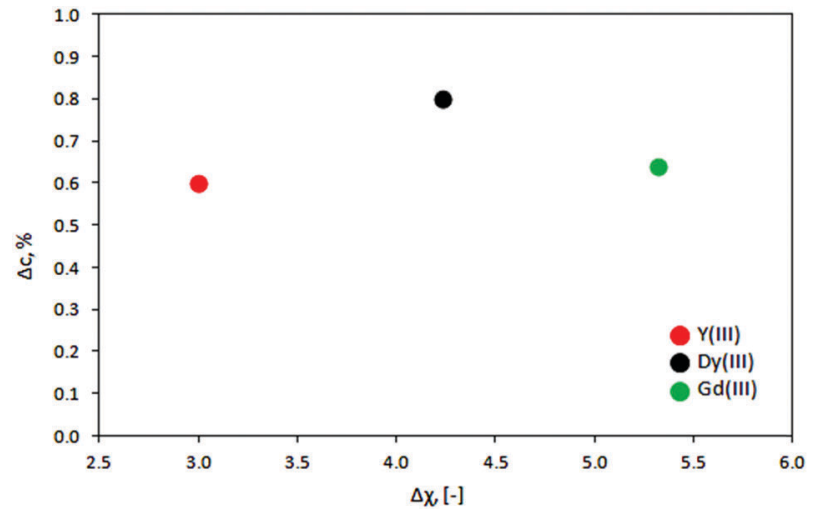

Fig. 9 Difference between the concentration of $\mathrm{Dy}^{3+}$ and $\mathrm{Gd}^{3+}$ ions in slice $A\left(0-2 \mathrm{~mm}\right.$ from the strongest magnetic field gradient point) and $\mathrm{Y}^{3+}$ ions in slice $\mathrm{C}$ (4-6 $\mathrm{mm}$ from the strongest magnetic field point) and their initial concentration in the gel samples after $1440 \mathrm{~min}$ of exposure to the magnetic field of the NdFeB magnets, as a function of difference between the dimensionless ([-]) magnetic susceptibilities of the migrating ions and the solvent.

results: the concentration changes of $\mathrm{Dy}^{3+}$ ions proceed twice as long as for $\mathrm{Mn}^{2+}$ ions, reaching the maximum enrichment twice lower than the one observed for $\mathrm{Mn}^{2+}$ ions in the same experimental setup. Hence, it was suspected that the induced magnetomigration of metal ions is not the migration of single ions, but a group of ions and other species present in solution, surrounding a core ion (cation), such as water molecules.

Fujiwara et $a .^{25-28}$ investigated the drift motion of metal ions spotted on silica gel and exposed to an inhomogeneous magnetic field. The authors concluded that the motion of ions in an external field is not a motion of single ions but a group of ions and water molecules with an estimated diameter of $2.4 \mu \mathrm{m}$. In this case, the magnetic energy acting on the formed group will be much higher than the one acting on a single ion. Gorobets et al. ${ }^{40}$ showed that ferrous ions formed due to the corrosion of a steel sphere in an external magnetic field follow a defined pattern in solution similar to the one of the magnetic field profile around the sphere. This was explained as a balance between the magnetic pressure and the osmotic pressure $n k_{\mathrm{B}} T$, where $n$ is the number of ions per unit volume, providing that the pressure is reduced by a factor of $10^{4}$, implying that ions respond collectively to a magnetic field. In this context, $\mathrm{Ca}^{2+}$ ions are now thought to form structured assemblies in water known as "dollops". ${ }^{24}$ However, the exact nature of this group formation is not clear yet and requires further investigations. Georgalis et al. ${ }^{41}$ suggested that it may be attributed to the direct electrostatic interactions between positive cations and negative counter ions, hydrophobic interactions or interaction between ions bearing identical charges caused by exclusion of ions by water molecules what might suggest that the magnetic field effect on ions and molecules in solution is closely related to the behavior of kosmotropic (structure-making) species.

\section{Conclusions}

Magnetic field gradients have a measurable effect on strongly paramagnetic $\mathrm{Dy}^{3+}$ and $\mathrm{Gd}^{3+}$, as well as on weakly diamagnetic 
$\mathrm{Y}^{3+}$ ions in solution. Paramagnetic ions move in the direction of increasing magnetic field, while diamagnetic ions move in the opposite direction. Even magnetic field gradients caused by strong NdFeB magnets are sufficient to induce sizeable concentration gradients in initially homogeneous solution of metal ions. Magnetic field gradients in a superconducting magnet of already $5 \mathrm{~T}$ led to concentration changes of the order of $10 \%$ in solutions containing $\mathrm{Dy}^{3+}$ ions. It was found that the concentration changes increase when the applied magnetic field gradient increases. The concentration changes however, do not change with the magnetic susceptibility of the ions, and the slightly diamagnetic $\mathrm{Y}^{3+}$ ions migrate almost as much under the same circumstances than the strongly paramagnetic $\mathrm{Dy}^{3+}$ and $\mathrm{Gd}^{3+}$ ions.

\section{Acknowledgements}

The authors would like to thank Johan Vanacken (Department of Physics and Astronomy, KU Leuven), Joop van Deursen (MTM, KU Leuven) and Avantes for their technical support. The authors thank the KU Leuven (projects IOF-KP RARE ${ }^{3}$ and GOA GOA/13/008) and the FWO Flanders (project G082716N) for financial support. The research leading to these results has received funding from the European Community's Seventh Framework Programme ([FP7/2007-2013]) under grant agreement no. 607411 (MC-ITN EREAN: European Rare Earth Magnet Recycling Network). This publication reflects only the author's view, exempting the Community from any liability. Project website: www.erean.eu.

\section{References}

1 R. Aogaki, T. Negishi, M. Yamato, E. Ito and I. Mogi, Physica $B, 1994,210,611$.

2 O. Aaboudi, J.-P. Chopart, J. Douglade, A. Olivier, C. Gabrielli and B. Tribollet, J. Electrochem. Soc., 1990, 137, 1796.

3 O. Devos, O. Aaboudi, J.-P. Chopart, E. Merienne, A. Oliver and J. Amblard, J. Electrochem. Soc., 1998, 145, 4139.

4 A. Oliver, J.-P. Chopart and J. Douglade, J. Electroanal. Chem., 1987, 217, 443.

5 G. Hinds, J. M. D. Coey and M. E. G. Lyons, J. Appl. Phys., 1998, 83, 6449.

6 G. Hinds, J. M. D. Coey and M. E. G. Lyons, Electrochem. Commun., 2001, 3, 218.

7 U. Schadewald, B. Halbedel, M. Ziolkowski and H. Brauer, International Scientific Colloquium, Modelling for Material Processing, Riga, 2010.

8 T. Weier, K. Eckert, S. Muhlenhoff, C. Cierpka, A. Bund and M. Uhlemann, J. Electrochem. Soc., 2007, 9, 2479.

9 G. Mutschke, K. Tschulik, T. Weier, M. Uhlemann, A. Bund and J. Frohlich, Electrochim. Acta, 2010, 55, 9060.

10 A.-L. Daltin, A. Addad, P. Baudart and J.-P. Chopart, CrystEngComm, 2011, 13, 3373.

11 M. Uhlemann, H. Schlorb, H. Msellak and J.-P. Chopart, J. Electrochem. Soc., 2004, 151, C598.
12 K. L. Rabah, J.-P. Chopart, H. Schloerb, S. Saulnier, O. Aaboubi, M. Uhlemann, D. Elmi and J. Amblard, J. Electroanal. Chem., 2004, 571, 85.

13 M. D. Pullins, K. M. Grant and H. S. White, J. Phys. Chem. B, 2001, 105, 8989.

14 M. Uhlemann, K. Tschulik, A. Gebert, G. Mutschke, J. Frohlich, A. Bund, X. Yang and K. Eckert, Eur. Phys. J.: Spec. Top., 2013, 220, 287.

15 P. Dunne, R. Soucaille, K. Ackland and J. M. D. Coey, Magnetohydrodyn., 2012, 48, 43.

16 D. J. Griffiths, Introduction to Electrodynamics, Benjamin Cummings, Inc., San Francisco, 3rd edn, 2008, ch. 6.

17 R. S. M. Rikken, R. J. M. Nolte, J. C. Maan, J. C. M. Van Hest, D. A. Wilson and P. C. M. Christianen, Soft Matter, 2014, 10, 1295.

18 R. P. Feynman, R. B. Leighton and M. L. Sands, The Feynman Lectures on Physics, Adison-Wesley, Reading, MA, 1964, vol. 2.

19 J. M. D. Coey, R. Aogaki, F. Byrne and P. Stamenov, Proc. Natl. Acad. Sci. U. S. A., 2009, 106, 8811.

20 P. Dunne and J. M. D. Coey, Phys. Rev. B: Condens. Matter Mater. Phys., 2012, 85, 224411.

21 M. C. Amiri and A. A. Dadkhah, Colloids Surf., A, 2006, 278, 252.

22 V. Kochmarsky, Magn. Electr. Sep., 1995, 7, 77.

23 P. Xiao-Feng and D. Bo, Phys. Rev. B: Condens. Matter Mater. Phys., 2008, 403, 3571.

24 R. Demichelis, P. Raiteri, J. D. Gale, D. Quigley and D. Gebauer, Nat. Commun., 2011, 2, 590.

25 M. Fujiwara, D. Kodoi, W. Duan and Y. Tanimoto, J. Phys. Chem. B, 2001, 105, 3343.

26 K. Chie, M. Fujiwara, Y. Fujiwara and Y. Tanimoto, J. Phys. Chem. B, 2003, 107, 14374.

27 M. Fujiwara, K. Chie, J. Sawai, D. Shimizu and Y. Tanimoto, J. Phys. Chem. B, 2004, 108, 3531.

28 M. Fujiwara, K. Mitsuda and Y. Tanimoto, J. Phys. Chem. B, 2006, 110, 13965.

29 X. Yang, K. Tschulik, M. Uhlemann, S. Odenbach and K. Eckert, J. Phys. Chem. Lett., 2012, 3, 3559.

30 B. Pulko, X. Yang, Z. Lei, S. Odenbach and K. Eckert, Appl. Phys. Lett., 2014, 105, 232407.

31 X. Yang, K. Tschulik, M. Uhlemann, S. Odenbach and K. Eckert, IEEE Trans. Magn., 2014, 11, 4600804.

32 Y. G. Galyametdinov, W. Haase, B. Goderis, D. Moors, K. Driesen, R. Van Deun and K. Binnemans, J. Phys. Chem. B, 2007, 111, 13881.

33 T. Takeda, T. Kogawa, I. Yamamoto and M. Yamaguchi, Proc. Int. Symp. Magneto-Science, 2006, 2, P34.

34 W. Noddack and E. Wicht, Phys. Chem., 1952, 56, 893.

35 I. Noddack and E. Wicht, Chem. Tech., 1955, 7, 3.

36 Landolt-Bornstein, Numerical Data and Functional Relationships in Science and Technology, New Series, II/16, ed. K.-H. Hellwege and A. M. Hellwege, Diamagnetic Susceptibility, Springer-Verlag, Heidelberg, 1986.

37 Landolt-Bornstein, Numerical Data and Functional Relationships in Science and Technology, New Series, III/19, Sub-volumes a 
to i2, W. Martienssen (Ed. in Chief), Magnetic Properties of Metals, Springer-Verlag, Heidelberg, 1986-1992.

38 Landolt-Bornstein, Numerical Data and Functional Relationships in Science and Technology, New Series, II/2, II/8, II/10, II/11, and II/12a, Coordination and Organometallic Transition Metal Compounds, Springer-Verlag, Heidelberg, 19661984.
39 G. Foëx, Tables de constantes et données numériques, Diamagnétisme et paramagnétisme, Relation paramagnétique, Masson, Paris, 1957, vol. 7.

40 O. Y. Gorobets, Y. I. Gorobets, I. A. Bondar and Y. A. Legenkiy, J. Magn. Magn. Mater., 2013, 330, 76.

41 Y. Georgalis, A. M. Kierzek and W. Saenger, J. Phys. Chem. B, 2000, 104, 3405. 